\title{
Chlorproguanil-Dapsone-Artesunate versus Chlorproguanil-Dapsone: A Randomized, Double-Blind, Phase III Trial in African Children, Adolescents, and Adults with Uncomplicated Plasmodium falciparum Malaria
}

\author{
Alfred B. Tiono,* Alassane Dicko, Dennis A. Ndububa, Tsiri Agbenyega, Simon Pitmang, Jacob Awobusuyi, \\ Allan Pamba, Stephan Duparc, Li-Ean Goh, $†$ Emma Harrell, Nick Carter, Stephen A. Ward, \\ Brian Greenwood, and Peter A. Winstanleył
}

Centre National de Recherche et de Formation sur le Paludisme, Ministère de la Santé, Ouagadougou, Burkina Faso; Malaria Research and Training Centre and Department of Public Health, Faculty of Medicine, Pharmacy and Dentistry, University of Bamako, Bamako, Mali; Obafemi Awolowo University Teaching Hospital, Department of Medicine, Ile Ife, Nigeria; Komfo Anokye Teaching Hospital, Kumasi, Ghana; Department of Medicine, Plateau State Specialist Hospital, Jos, Plateau State, Nigeria; Lagos State University Teaching Hospital, Department of Medicine, Ikeja, Lagos, Nigeria; GlaxoSmithKline, Greenford, Middlesex, United Kingdom; GlaxoSmithKline, Harlow, Essex; Liverpool School of Tropical Medicine, Liverpool, United Kingdom; Department of Infectious and Tropical Diseases, London School of Hygiene and Tropical Medicine, London, United Kingdom; School of Clinical Sciences, University of Liverpool, Liverpool, United Kingdom

\begin{abstract}
This multi-center, randomized, parallel-group, double-blind, double-dummy study compared the efficacy and safety of chlorproguanil-dapsone-artesunate (CDA) and chlorproguanil-dapsone (CPG-DDS) in the treatment of falciparum malaria in Africa (Burkina Faso, Ghana, Mali, Nigeria). Six hundred patients ( $\geq 1$ year of age) received CDA 2.0/2.5/4.0 mg/kg, and 292 CPG-DDS $2.0 / 2.5 \mathrm{mg} / \mathrm{kg}$, once daily for 3 days. Day 28 parasitologic cure rate (polymerase chain reaction [PCR]-corrected, per-protocol population) was $89.1 \%(416 / 467)$ for CDA, non-inferior but also superior to CPG-DDS, $83.0 \%(176 / 212)$ (treatment difference $6.1 \%$; $95 \%$ confidence interval [CI] 0.3, 11.9). Glucose-6-phosphate dehydrogenase (G6PD) genotype was available for 844/892 (95\%) patients. Occurrences of a composite hemoglobin safety endpoint (hemoglobin drop $\geq 40 \mathrm{~g} / \mathrm{L}$ or $\geq 40 \%$ versus baseline, hemoglobin $<50 \mathrm{~g} / \mathrm{L}$, or blood transfusion) were CDA 13/44 (30\%), CPG-DDS 7/24 (29\%) in G6PD-deficient patients versus CDA 4/448 (<1\%), CPG-DDS 6/221 (3\%) in G6PD-normal patients. No deaths occurred. CDA was more efficacious than CPG-DDS. However, the hemolytic potential in G6PD-deficient patients does not support further development of CDA.
\end{abstract}

\section{INTRODUCTION}

There were over 213 million clinical malaria episodes in subSaharan Africa in 2006, causing over 800,000 deaths, mostly in children less than 5 years of age. ${ }^{1}$ Although recent progress has been made in addressing the malaria burden in Africa, ${ }^{2}$ the efficacy of the most affordable anti-malarials has been compromised by the widespread emergence of Plasmodium falciparum resistance to these agents. ${ }^{1}$

Chlorproguanil-dapsone (CPG-DDS,Lapdap ${ }^{\mathrm{TM}}$ ) was developed through a public-private partnership as a low-cost, fixeddose combination for the treatment of uncomplicated malaria caused by $P$. falciparum. ${ }^{3}$ Studies of CPG-DDS efficacy and safety were encouraging and the file was approved by the UK Medicines and Healthcare Products Regulatory Agency in 2003. In a pivotal study of 1,850 children, CPG-DDS had Day 14 cure rates of $93-99 \%$ and was more effective than sulfadoxinepyrimethamine (SP) in three of five African countries. ${ }^{4}$

Artemisinin derivatives are active against multidrug-resistant $P$. falciparum, ${ }^{5,6}$ rapidly reduce asexual parasitemia,, 8 and are gametocytocidal, potentially reducing transmission. ${ }^{9-13}$ However, the short half-life of these agents precludes their use as monotherapy for uncomplicated malaria. To obtain the

\footnotetext{
*Address correspondence to Alfred B. Tiono, Centre National de Recherche et de Formation sur le Paludisme Ministère de la Santé, 01 BP 2208, Ouagadougou 01, Burkina Faso. E-mail: t.alfred@fasonet.bf $\dagger$ Deceased.

$\ddagger$ Contributors: The study team also consisted of the following coinvestigators: Alphonse Ouedraogo (Ouagadougou, Burkina Faso); Daniel Ansong and Emmanuel Asafo-Adjei (Kumasi, Ghana); Ousmane Guindo and Ousmane Kante (Doneguebougou and Banambani, Mali); Adesola O. Oyelese and Norah O. Akinola (Ile-Ife, Nigeria); Comfort A. Daniyam and Ibrahim Pate (Jos, Nigeria); and Mohammed Salisu and Ayodele Brodie-Mend (Lagos, Nigeria).
}

advantages of artemisinins, the World Health Organization (WHO) Global Malaria Program recommends artemisininbased combination therapy (ACT) for first-line treatment of P. falciparum malaria in Africa. ${ }^{14}$ Chlorproguanil-dapsoneartesunate (CDA) was, therefore, developed as a low-cost, simple, fixed-dose ACT for use in Africa. Phase I and II studies were promising, with good efficacy and a safety profile consistent with $\mathrm{CPG}-\mathrm{DDS} .{ }^{15}$

Dapsone has been used extensively in leprosy and is known to reduce erythrocyte lifespan. This may be observed as hemolysis and methemoglobinemia, though these have generally been considered mild and self limiting. A clinical safety trial of CPG-DDS suggested that the hemolytic effect of dapsone might be compounded in malaria patients with glucose-6phosphate dehydrogenase (G6PD) deficiency, though data were insufficient to fully assess the clinical significance of the findings. ${ }^{4,16}$ G6PD deficiency is X-linked, therefore more common in males. ${ }^{17}$ In hemizygous males, the most common African variant, G6PD A-, results in 10-15\% of normal enzyme activity. ${ }^{17}$ Female heterozygotes display mosaicism, with varying degrees of phenotypic enzyme activity.$^{18}$ When exposed to oxidative stress, G6PD-deficient erythrocytes become damaged and are removed from circulation by the spleen. ${ }^{17}$ Otherwise, G6PD-deficient patients are asymptomatic. ${ }^{17}$ G6PD deficiency is common in populations exposed to malaria, and it may provide some protection from severe malaria. ${ }^{17}$

This Phase III randomized clinical trial was designed to compare the efficacy and safety of CDA and CPG-DDS. A second Phase III trial of similar design compared CDA with artemether-lumefantrine (AL) and has been published elsewhere. ${ }^{19}$ Both trials used parasitologic cure (corrected for reinfection) at Day 28 as the primary efficacy outcome, as this was the standard WHO-recommended efficacy outcome at the time the studies were designed. This study also thoroughly 
investigated the possibility of G6PD-related hemolysis in patients treated with CDA and CPG-DDS.

\section{METHODS}

This randomized, parallel-group, double-blind, doubledummy study was conducted between April 2006 and May 2007 at seven sites in four African countries: Burkina Faso (Ouagadougou), Ghana (Kumasi), Mali (Doneguebougou and Banambani), and Nigeria (Ile-Ife, Jos, and Lagos). Ethical approval was obtained from each participating center's ethics committee or institutional review board and the WHO. Study procedures were conducted in accordance with Good Clinical Practice, applicable regulatory requirements, and the Declaration of Helsinki. The study was overseen by an Independent Data Monitoring Committee (IDMC), which appointed an independent end-point reviewer. Two IDMC interim safety analyses were planned.

Patients. Eligible subjects were males or females, $\geq 1$ year of age, with acute uncomplicated $P$. falciparum malaria with 2,000-200,000 parasites $\mu \mathrm{L}^{-1}$. Inclusion criteria at screening were history of fever within $24 \mathrm{hr}$ or tympanic temperature $\geq 37.5^{\circ} \mathrm{C}$; weight $\geq 7.5 \mathrm{~kg}$; hemoglobin $\geq 70 \mathrm{~g} / \mathrm{L}$ or hematocrit $\geq 25 \%$ (if hemoglobin not available); ability and willingness to comply with the protocol. Written or oral witnessed consent was obtained from all study participants or their parents/ guardians. Assent was also required for subjects who were 12-18 years of age.

Patients were excluded from the study if they had features of severe/complicated $P$. falciparum malaria; evidence of concomitant infection (including Plasmodium vivax, Plasmodium ovale, or Plasmodium malariae); any underlying disease that might compromise malaria diagnosis or evaluation of the response to study medication (including clinical symptoms of immunosuppression, tuberculosis, bacterial infection, cardiac or pulmonary disease); known hypersensitivity to study drugs or their excipients or allergy to biguanides, sulphones, sulphonamides, or artemisinin derivatives; known G6PD deficiency, methemoglobin-reductase deficiency, hemoglobin $\mathrm{M}$ or $\mathrm{E}$, or porphyria; neonatal hyperbilirubinemia; malnutrition (defined as a weight:height ratio $<-3$ standard deviations or $<70 \%$ of the median of the National Center for Health Statistics/WHO normalized reference values); previously participated in the study; received any medications with hemolytic potential; or received recent anti-malarial therapy that would affect the efficacy evaluation or any investigational drug within 30 days or five half-lives (whichever longer). Female patients with a positive pregnancy test, or who refused consent for a pregnancy test, or who were breastfeeding were also excluded.

Objectives. This study evaluated the efficacy of CDA versus CPG-DDS in uncomplicated $P$. falciparum malaria, employing a non-inferiority comparison of parasitologic cure at Day 28 corrected for re-infection. This study also sought to further qualify the safety of CDA versus CPG-DDS; a 2:1 CDA:CPGDDS randomization schedule was used to maximize the safety information obtained for CDA.

Study procedures. Eligible subjects were randomized to CDA 2/2.5/4 mg/kg/day (GlaxoSmithKline, Greenford, UK) or CPG-DDS $2 / 2.5 \mathrm{mg} / \mathrm{kg} /$ day (GlaxoSmithKline), once daily for 3 days (Days 0,1 , and 2). For younger children, tablets were crushed and mixed with water just before administration. All therapy was directly observed. Patients vomiting within $30 \mathrm{~min}$ of dosing could be re-dosed. Vomiting again within $30 \mathrm{~min}$ of re-dosing led to administration of rescue medication (as per local clinical practice guidelines) and the subject was followed for safety assessment until Day 28. Enrolled patients were seen on Days 0,1,2, and 3 as outpatients, with clinic follow-up assessments at Days 7,14, and 28, plus additional visits if thought necessary by the investigator or the patient. A home visit by a trained fieldworker was also conducted on Days 4, 5 , and 6 to check that the patient was well enough to remain at home.

Laboratory procedures. Blood samples $(\sim 10 \mu \mathrm{L})$ for parasite count assessment were taken by thumb prick at screening, before dosing on Days 0,1, and 2 and on Days 3, 7, 14, and 28 plus any additional visits after Day 28. Thick films were Giemsa stained and parasites enumerated independently by two microscopists blinded to study treatment. Parasite densities were calculated according to WHO protocolWHO/HTM/RBM/2003.50. ${ }^{20}$ Gametocyte densities were read from thick films at screening, Day 0 (pre-dose) and Days 1, 2, 3, 7, 14, and 28 against a minimum of 200 white blood cells (WBCs).

Two drops of blood were stored on filter paper for polymerase chain reaction (PCR) analysis of parasite genotype using $m s p-1, m s p$-2, and glurp. ${ }^{21,22}$ For patients with clinical or parasitologic failure after Day 7, parasite genotype at Day 0 (pre-dose) versus Day of failure were compared in order to distinguish re-infection from recrudescence. Patients with indeterminate PCR results were considered to have missing data for that time point.

For G6PD genotype analysis, two drops of blood were collected at Day 0 (pre-dose) onto pre-printed filter papers. The G6PD genotyping was performed by two central laboratories (Shoklo Malaria Research Unit, Mae Sot, Thailand and Kenya Medical Research Institute, Nairobi, Kenya). Following DNA extraction, PCR primers were used for amplification of loci at $376 \mathrm{~A} \rightarrow \mathrm{G}, 202 \mathrm{G} \rightarrow \mathrm{A}, 542 \mathrm{G} \rightarrow \mathrm{T}, 680 \mathrm{G} \rightarrow \mathrm{T}$, and 968 $\mathrm{T} \rightarrow \mathrm{C}^{18,23-25}$ Amplicons were analyzed using restriction fragment length polymerization, allowing recognition of wildtype G6PD B, and the common African mutations G6PD A, and the G6PD-deficient mutation A-. ${ }^{18,23-25}$ The G6PD phenotype was determined at a central laboratory (Synexa, Cape Town, South Africa) from a Day 0 (pre-dose) blood sample using a commercial NADPH fluorescence test (Trinity Biotech, Wicklow, Ireland). Phenotypes were defined versus controls as normal, intermediate, and deficient for G6PD activity. The test distinguishes normal/intermediate G6PD enzyme activity from grossly G6PD-deficient samples.

Venous blood $(2 \mathrm{~mL})$ samples for hematology evaluations were taken at screening and at Days 1,2,3,7,14, and 28. Samples for clinical chemistry were taken at Days 0 (pre-dose), 3, 7, and 28 and at Day 14 if Day 7 values were abnormal.

Efficacy assessment. The primary efficacy endpoint, PCRcorrected parasitologic cure rate, was defined as clearance of the initial malaria infection by Day 7 with the subject remaining infection free at Day 28. Day 14 parasitologic cure was defined as clearance of the initial malaria infection by Day 7, with the subject remaining infection-free at Day 14. The key secondary endpoint was the proportion of subjects with parasites remaining $24 \mathrm{hr}$ after the first treatment dose. The reduction in parasite load versus baseline $24 \mathrm{hr}$ after the first treatment dose was also calculated.

Other secondary endpoints assessed throughout the study were geometric mean asexual parasite densities; percent 
reduction from baseline in parasite load; the proportion of subjects with gametocytes; gametocyte densities; presence (and severity) of the signs and symptoms of malaria; and body temperature. Derived fever clearance time was defined as the time (in hours) after the first dose of study medication to the time when body temperature decreased to $<37.5^{\circ} \mathrm{C}$, and remained so for at least $48 \mathrm{hr}$. Adequate clinical and parasitologic response (ACPR) and PCR-corrected ACPR (ACPRp), excluding patients with new infections, were determined for Days 14 and 28. ACPR(p) was defined per WHO (2003) criteria as, absence of parasitemia, irrespective of tympanic temperature, without previous treatment failure. ${ }^{20}$

Safety assessment. Adverse events were recorded at screening, pre- and post-treatment on Day 0 and at all subsequent study visits and classified using the Medical Dictionaries for Regulatory Activities (MedDRA) coding system (Version 10.1). Treatment-emergent adverse events were defined as any unfavorable and unintended sign, symptom, or disease (new or exacerbated) temporally associated with the use of a medicinal product. A serious adverse event was defined as resulting in death (or life-threatening); hospitalization or prolonged hospitalization; disability or incapacity; a congenital or birth defect; or was deemed serious by the investigator. Additional serious adverse event criteria were a decrease in hemoglobin of $\geq 40 \%$ from baseline; blood transfusion; a hemoglobin concentration of $<50 \mathrm{~g} / \mathrm{L}$; methemoglobin $\geq 20 \%$; and methemoglobin $\geq 10-20 \%$ with clinical symptoms of methemoglobinemia.

Hemolytic effect was assessed using a composite "hemoglobin safety" endpoint, defined prospectively as a hemoglobin decrease of $\geq 40 \mathrm{~g} / \mathrm{L}$ or $\geq 40 \%$ versus baseline, or hemoglobin $<$ $50 \mathrm{~g} / \mathrm{L}$, or blood transfusion.

Sample size. For the primary endpoint analysis, 420 evaluable subjects in the CDA group and 210 in the CPG-DDS group were needed to provide at least $90 \%$ power to show noninferiority of CDA to CPG-DDS with a one-sided hypothesis test and a $2.5 \%$ significance level. This calculation was based on a $7 \%$ non-inferiority margin, a 2:1 allocation ratio, and $93 \%$ efficacy for both treatments. Allowing for a $30 \%$ data loss, target randomization was 600 to CDA and 300 to CPG-DDS.

Randomization and blinding. The computer-generated randomization schedule was provided by GlaxoSmithKline. Patients were allocated to a treatment group by the Registration and Medication Ordering System (RAMOS), accessed by telephone. In case of emergency, the investigator could unblind a subject's treatment by RAMOS. Treatment groups were stratified according to age: 1 to $<5$ years; 5 to $<15$ years; and $\geq 15$ years to achieve at least $10 \%$ of target recruitment into each age category, the remaining allocation being unrestricted. All site investigators, laboratory personnel, and GlaxoSmithKline were blinded to patient allocation. Matching placebo was provided for both active treatments.

Statistical procedures. The intent-to-treat (ITT) population included all randomized patients who received at least one dose of study medication. The per-protocol (PP) population was a sub-set of the ITT population, including those patients who did not violate the protocol to the extent that could impact the efficacy analysis.

In the ITT population, patients without a Day 28 parasitologic assessment were considered treatment failures for the primary endpoint. For the PP population, subjects with missing data were excluded from the relevant analyses. For both the PP and ITT analyses, patients with PCR-determined new infections were considered treatment successes at that time point. At subsequent time points, these patients were considered missing for the PP analysis and failures in the ITT analysis.

The null hypothesis was that CDA efficacy was inferior to that of CPG-DDS based on a primary endpoint of Day 28 PCR-corrected parasitologic cure rate in the PP population. Two-sided $95 \%$ confidence intervals (CI) were calculated for this endpoint using the normal approximation to the binomial distribution. Non-inferiority was shown if the lower limit of the $95 \%$ CI (CDA minus CPG-DDS) was equal to or greater than $-7 \%$. No adjustments for multiple comparisons were required. The effects of center, age group, and baseline parasitaemia count (categorized by $\leq 33 \%$ quantile, $>33 \%-$ $<67 \%$ quantile, and $\geq 67 \%$ quantile) were studied using logistic regression. The ITT population was used for a supportive analysis of the primary endpoint. A sensitivity analysis was performed on parasitologic cure for the Day 28 ITT population using observed cases only, i.e. patients with missing data were excluded rather than treated as failures.

For the key secondary endpoint, the proportion of subjects with parasites present at $24 \mathrm{hr}$ after the initial dose of study medication, treatment groups were compared using a $\chi^{2}$ test at a two-sided $5 \%$ significance level using the ITT population. The ACPR and ACPRp were evaluated using the ITT and PP populations. The PP population was analyzed for reduction in parasite load versus baseline $24 \mathrm{hr}$ after the first treatment dose. All other secondary efficacy endpoints and safety analyses were analyzed using the ITT population.

Adverse events, serious adverse events, and laboratory abnormalities were tabulated by treatment group and summarized using descriptive statistics. The frequency of serious adverse events, hematologic adverse events and hemoglobin data, and occurrences of the hemoglobin safety endpoint were analyzed by G6PD status, using the following terms: G6PD normal, G6PD heterozygous females, and G6PD deficient (A- hemizygous males plus A-/A- homozygous females). Occurrence of the hemoglobin safety composite endpoint was analyzed using logistic regression modeling to determine the contribution of G6PD genotype, weight, age, baseline hemoglobin, baseline parasitaemia, and treatment. Hemoglobin changes from baseline to Day 7 were studied using analysis of variance, with terms for treatment, age, initial parasitemia, and actual dapsone dose (expressed as $\mathrm{mg} / \mathrm{kg}$ ), and expressed as treatment difference and 95\% CI. Statistical analyses were performed by GlaxoSmithKline using SAS (version 8.2, SAS Institute Inc., Cary, NC).

\section{RESULTS}

Patients. The study population included 892 randomized subjects, all of whom received study treatment (ITT population): 600 received CDA and 292 CPG-DDS (Figure 1). The proportion of patients that withdrew prematurely from the study was similar between treatment groups (Figure 1). Demographic and baseline clinical characteristics of the ITT population were similar for the two treatment groups (Table 1). Overall, mean age $( \pm \mathrm{SD})$ was $7.3 \pm 9.4$ years, mean weight $20.8 \pm 14.9 \mathrm{~kg}, 49 \%$ of patients were male, and all patients were black African. The G6PD genotype was available for 844/892 (95\%) patients, of whom 53/414 (13\%) were A- hemizygous 


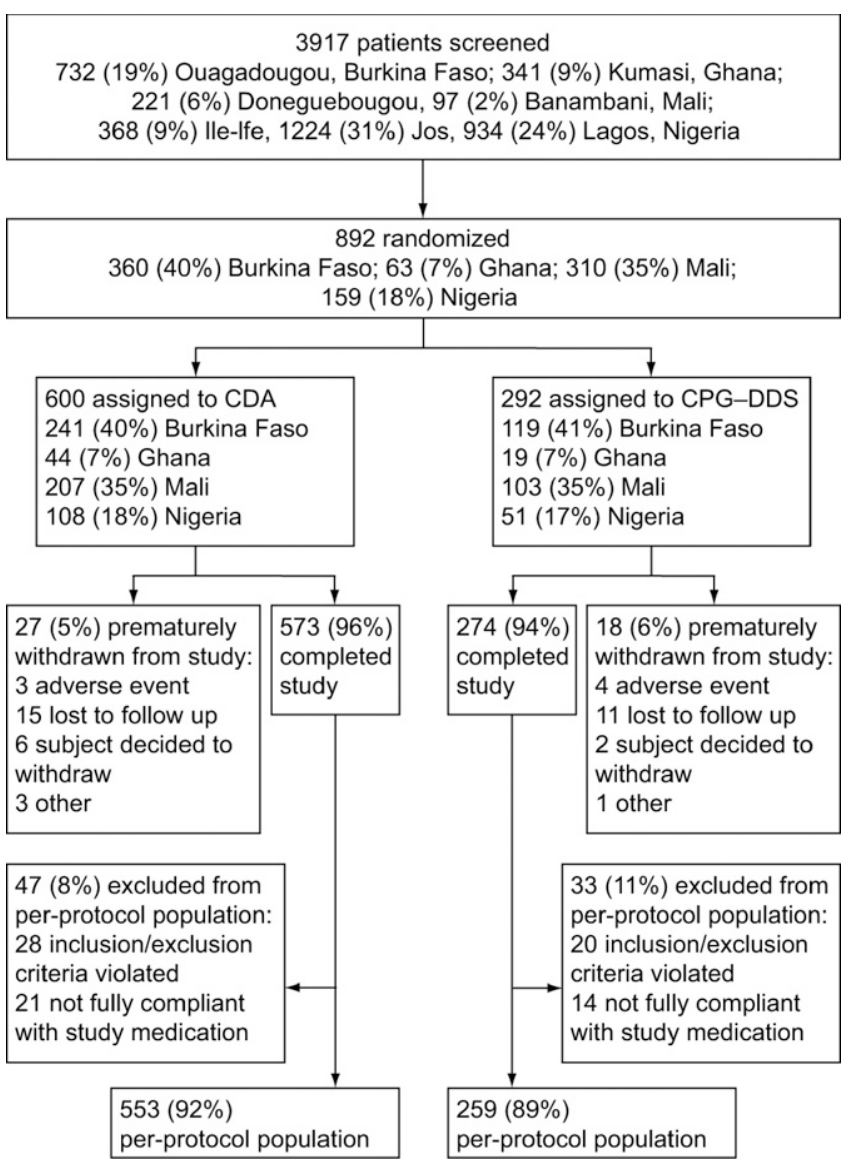

FIGURE 1. Trial profile. NB: There may have been more than one reason for a patient's withdrawal from the study or exclusion from the PP population.

males and 15/430 (3\%) A-/A- homozygous females (i.e., G6PD deficient, Table 2). Excluding patients with missing G6PD genotype data, Ile Ife had the highest proportion of G6PD-deficient subjects (11.3\% [12/106]) and Jos the lowest $(0 / 21)$. For hemizygous males, the highest proportion was $15.5 \%$ (27/174) from Ouagadougou and the lowest was Jos $(0 / 10)$. Given this large range, not surprisingly the proportion of female genotypes was significantly different from expected values using the Hardy-Weinberg equation. G6PD phenotype was available for $747 / 892(84 \%)$ patients (Table 2). In male patients, concordance between G6PD genotype and phenotype (i.e., both were normal or both deficient) was $89 \%$ (321/361). Baseline characteristics of the PP population were similar to those of the ITT population. Treatment compliance (patients receiving all doses at the correct dose) was 96\% (576/600) for CDA and 95\% (276/292) for CPG-DDS.

Efficacy. The PCR-corrected parasitologic cure at Day 28 in the PP population (primary efficacy endpoint) was $89.1 \%$ for CDA versus $83.0 \%$ for CPG-DDS (treatment difference $6.1 \%$; $95 \%$ CI $0.3,11.9$ ) (Table 3). Thus, CDA was both noninferior and superior to CPG-DDS for this endpoint. Logistic regression analysis of the primary endpoint found no effect of center, age, or baseline parasitemia, but the CPG-DDS:CDA odds ratio (OR) of $0.6(95 \%$ CI $0.37,0.97 ; P=0.037)$ for treatment success implied superiority for CDA. Outcomes for the ITT analysis were supportive of the PP analysis (Table 3). A sensitivity analysis using observed data only found similar results, with PCR-corrected parasitologic cure rates of 438/498 (88.0\%) with CDA and 184/235 (78.3\%) with CPG-DDS (treatment difference 9.7\%; 95\% CI 3.7, 15.6). Parasitologic cure at Day 14 was also non-inferior and superior with CDA versus CPG-DDS (Table 3).

Asexual parasite count decreased rapidly from baseline in both treatment groups during the first $24 \mathrm{hr}$ following the start of therapy. After $24 \mathrm{hr}$ of therapy, for the ITT population, significantly fewer patients in the CDA group had parasites remaining (288/600 [48.0\%]) versus the CPG-DDS group (259/292 [88.7\%], treatment difference $-40.7 ; 95 \%$ CI -46.1 , $-35.3 ; P<0.001$ ) (Figure 2A). The mean reduction in parasite load versus baseline $( \pm \mathrm{SD}) 24 \mathrm{hr}$ after therapy start was $99.6 \pm 1.9 \%$ with CDA and $84.1 \pm 84.4 \%$ for $\mathrm{CPG}-\mathrm{DDS}$ in the PP population.

For those patients with a baseline temperature $\geq 37.5^{\circ} \mathrm{C}$, in the CDA group, 243/394 (62\%; 95\% CI 59.6, 66.5) patients had fever clearance within $24 \mathrm{hr}$ versus $61 / 199$ (31\%; $95 \%$ CI 24.3 , 37.1 ) in the CPG-DDS group. Mean body temperature normalized more rapidly in the CDA versus CPG-DDS group (Figure $2 \mathrm{~B})$. Mean $( \pm \mathrm{SD})$ time to fever clearance was $33.1 \pm$ $20.1 \mathrm{hr}$ in the CDA group and $45.7 \pm 21.4 \mathrm{hr}$ for CPG-DDS.

TABLE 1

Demographic and baseline clinical characteristics (ITT population)*

\begin{tabular}{lll}
\hline \multicolumn{1}{c}{ Characteristic } & \multicolumn{1}{c}{ CDA $(N=600)$} & CPG-DDS $(N=292)$ \\
\hline Female, $\mathrm{n}(\%)$ & $293(49)$ & $160(55)$ \\
Age, years, mean $(\mathrm{SD})$ [range] & $7.4(9.5)[1-72]$ & $7.1(9.3)[1-72]$ \\
$1-<5, \mathrm{n}(\%)$ & $305(51)$ & $146(50)$ \\
$5-<15, \mathrm{n}(\%)$ & $234(39)$ & $115(39)$ \\
$\geq 15, \mathrm{n}(\%)$ & $61(10)$ & $31(11)$ \\
Weight, $\mathrm{kg}$, mean $(\mathrm{SD})$ [range] & $21.0(15.0)[8-112]$ & $20.4(14.9)[8-76]$ \\
Parasitemia, $\mu \mathrm{L}^{-1}$, geometric mean [range] & $21640[988-200,000]$ & $25573[576-200,000]$ \\
Temperature, ${ }^{\circ} \mathrm{C}$, mean $(\mathrm{SD})$ [range] & $37.9(1.0)[35.0-40.7]$ & $38.0(1.0)[35.5-40.2]$ \\
Patients with fever $\left(\geq 37.5^{\circ} \mathrm{C}\right), \mathrm{n}(\%)$ & $394(66)$ & $199(68)$ \\
Hemoglobin, $\mathrm{g} / \mathrm{L}$, mean $(\mathrm{SD})[$ range] & $104.0(17.1)[45.0-158.0]$ & $103.3(16.2)[66.0-161.0]$ \\
1 to $<5$ years & $95.1(14.2)[45.0-139.0]$ & $94.6(13.0)[66.0-126.0]$ \\
5 to $<15$ years & $109.6(12.8)[70.0-142.0]$ & $108.46(11.8)[75.0-143.0]$ \\
$\geq 15$ years & $127.0(14.2)[93.0-158.0]$ & $125.4(15.1)[93.0-161.0]$ \\
Hematocrit, $\mathrm{L}$, mean $(\mathrm{SD})$ & $0.314(0.051)$ & $0.313(0.047)$ \\
Red blood cell count $\times 10^{12} / \mathrm{L}$, mean $(\mathrm{SD})$ & $3.99(0.63)$ & $4.00(0.57)$ \\
Platelet count $\times 10^{9} / \mathrm{L}, \mathrm{mean}(\mathrm{SD})$ & $202.4(98.5)$ & $208.9(125.2)$ \\
White blood cell count $\times 10^{\%} / \mathrm{L}$, mean $(\mathrm{SD})$ & $9.75(4.24)$ & $10.51(4.54)$ \\
\hline
\end{tabular}

* ITT $=$ intent-to-treat CDA $=$ chlorproguanil-dapsone-artesunate CPG-DDS = chlorproguanil-dapsone. 
TABLE 2

G6PD status by genotype and phenotype (ITT population)*

\begin{tabular}{llccc}
\hline Category, n (\%) & $\begin{array}{c}\text { Genotype/ } \\
\text { phenotype } \dagger\end{array}$ & CDA & CPG-DDS & Total \\
\hline Males & & $(N=307)$ & $(N=132)$ & $(N=439)$ \\
Missing & & $19(6)$ & $5(4)$ & $24(5)$ \\
Genotype: & A & $288(93)$ & $127(96)$ & $415(95)$ \\
& B & $165(30)$ & $39(31)$ & $124(30)$ \\
& A- & $38(13)$ & $73(57)$ & $238(57)$ \\
Females & & $(N=293)$ & $(N=160)$ & $(N 3(13)$ \\
Missing & & $19(6)$ & $5(3)$ & $24(5)$ \\
Genotype: & A/A & $274(94)$ & $155(97)$ & $429(95)$ \\
& A/B & $77(14)$ & $10(6)$ & $47(11)$ \\
& B/B & $87(32)$ & $35(23)$ & $109(25)$ \\
& A/A- & $16(6)$ & $11(7)$ & $151(35)$ \\
& B/A- & $54(20)$ & $26(17)$ & $80(19)$ \\
& A-/A- & $6(2)$ & $9(6)$ & $15(3)$ \\
All patients & & $(N=600)$ & $(N=292)$ & $(N=892)$ \\
Missing & & $99(17)$ & $46(16)$ & $145(16)$ \\
Phenotype: & & $501(84)$ & $246(84)$ & $747(84)$ \\
& Normal & $406(81)$ & $202(82)$ & $608(81)$ \\
& Intermediate & $47(9)$ & $26(11)$ & $73(10)$ \\
& Deficient & $48(10)$ & $18(7)$ & $66(9)$ \\
\hline
\end{tabular}

${ }^{*}$ ITT $=$ intent-to-treat; $\mathrm{CDA}=$ chlorproguanil-dapsone-artesunate; $\mathrm{CPG}-\mathrm{DDS}=$ chlorproguanil-dapsone.

$\dagger$ Percentages for genotype and phenotype exclude missing data.

In most patients, other malaria signs and symptoms had resolved by Day 3 in both treatment groups, with no remarkable differences between study therapies.

At baseline, gametocytes were present in 33/567 (6\%) patients in the CDA group and 13/279 (4\%) in the CPG-DDS group (ITT population). In the CDA group, the proportion of patients with gametocytes remained stable during treatment and declined during follow-up, whereas in the CPG-DDS group, the proportion increased during therapy and reached a maximum of $24 \%$ during follow-up (Day 7) (Figure 2C). Mean geometric gametocyte count was $1.2 \mu \mathrm{L}^{-1}$ in both treatment groups at baseline. In the CDA group, gametocyte count remained between $1.1-1.3 \mu \mathrm{L}^{-1}$ throughout therapy and follow-up. In the CPG-DDS group, gametocyte count increased to a maximum of $3.0 \mu \mathrm{L}^{-1}$ at Day 7 , decreasing to $1.4 \mu \mathrm{L}^{-1}$ at Day 28 (Figure 2C).

Day 28 ACPRp was $89 \%$ with CDA and $79 \%$ with CPGDDS, with $2 \%$ and $11 \%$ early treatment failures, respectively (Table 4). By Day $28,32 \%$ of patients receiving CDA and $30 \%$ receiving CPG-DDS in the PP population had experienced a re-infection (Table 4). Results for the ITT population showed similar trends (Table 4).
Adverse events. Treatment-emergent adverse events were reported for 38\% (227/600) of CDA-treated and 49\% (144/292) of CPG-DDS-treated patients. The most frequently occurring $(\geq 2 \%$ in either arm) adverse events included (CDA, CPGDDS): malaria (13\%,15\%); pyrexia $(6 \%, 10 \%)$; cough $(4 \%, 4 \%)$; vomiting $(3 \%, 5 \%)$; diarrhea $(3 \%, 1 \%)$; pneumonia $(3 \%, 2 \%)$; abdominal pain $(1 \%, 3 \%)$; and respiratory tract infection $(1 \%, 3 \%)$. Furthermore, there were 18 (3\%) adverse events potentially related to oxidative hemolysis in the CDA group (14 anemia, four decreased hemoglobin) and 12 (4\%) in the CPG-DDS group (nine anemia, two decreased hemoglobin, one intravascular hemolysis).

Twenty-one serious adverse events occurred in 16/600 (3\%) patients in the CDA group and 13 occurred in 12/292 (4\%) patients in CPG-DDS group. Anemia (CDA 11 cases, CPGDDS six cases) and decreased hemoglobin (CDA four cases, CPG-DDS two cases) were the most frequent serious adverse events.

There were no deaths in the study. Early withdrawals from the study for adverse events occurred in $3(<1 \%)$ patients receiving CDA and $6(2 \%)$ receiving CPG-DDS. In all but one case, the reason for withdrawal was nausea/vomiting. The remaining case in the CPG-DDS group (G6PD genotype missing) had intravascular hemolysis and (unconfirmed) sepsis. All adverse events leading to withdrawal occurred during the dosing period (Days 0-2).

Hemoglobin safety. The composite hemoglobin safety endpoint occurred most frequently in G6PD-deficient patients: $13 / 44(30 \%)$ in the CDA group and 7/24 (29\%) in the CPGDDS group (Table 5). Logistic regression analysis of potential risk factors for occurrences of the hemoglobin safety endpoint found a highly significant effect of G6PD deficiency versus G6PD normal: OR 40.0 (95\% CI 16.1, 99.4; $P<0.001)$. There was no difference between G6PD heterozygous versus G6PD normal, between the two study treatments, or for the other co-factors: weight, age, baseline hemoglobin, or parasitemia.

Five subjects required blood transfusions: two in the CDA group (one G6PD hemizygous A- male and one G6PD normal male) and three in the CPG-DDS group (one G6PD hemizygous A- male, one G6PD homozygous A-/A- female and one with unknown G6PD genotype and phenotype).

Hematologic laboratory data. Mean hemoglobin concentration decreased from baseline in both treatment groups, with no significant differences between treatment groups (Figure 3A). For CDA, hemoglobin reached a nadir on Day 7 of $93.2 \mathrm{~g} / \mathrm{L}$ versus $94.0 \mathrm{~g} / \mathrm{L}$ for CPG-DDS. For CPG-DDS, the minimum hemoglobin concentration occurred at Day 3;

TABLE 3

Parasitologic cure rates. PCR-corrected parasitologic cure at Day 28 (PP population) was the primary efficacy endpoint*

\begin{tabular}{|c|c|c|c|c|c|}
\hline \multirow[b]{2}{*}{ Analysis } & \multirow[b]{2}{*}{ Parasitologic cure rate, $\mathrm{n} / N(\%)$} & \multicolumn{2}{|c|}{ PP population $\dagger$} & \multicolumn{2}{|c|}{ ITT population } \\
\hline & & $\mathrm{CDA}$ & CPG-DDS & CDA & CPG-DDS \\
\hline \multirow[t]{2}{*}{ Day 14} & PCR corrected & $528 / 537(98.3)$ & $235 / 249(94.4)$ & $558 / 600(93.0)$ & $252 / 292(86.3)$ \\
\hline & Treatment difference, $\%$ (95\% CI) & \multicolumn{2}{|c|}{$3.9(0.9,7.0)$} & \multicolumn{2}{|c|}{$6.7(2.3,11.1)$} \\
\hline Day 28 & $\begin{array}{l}\text { PCR corrected } \\
\text { Treatment difference, \% }(95 \% \mathrm{CI})\end{array}$ & $416 / 467(89.1)$ & .9) & $\begin{array}{r}438 / 600(73.0) \\
10 .\end{array}$ & $\begin{array}{l}184 / 292(63.0) \\
6.6)\end{array}$ \\
\hline
\end{tabular}

* $\mathrm{PCR}=$ polymerase chain reaction $; \mathrm{PP}=$ per-protocol; $\mathrm{ITT}=$ intent-to-treat CDA = chlorproguanil-dapsone-artesunate; $\mathrm{CPG}-\mathrm{DDS}=$ chlorproguanil-dapsone CI $=$ confidence interval.

$\dagger$ In the PP analysis, patients with missing data were excluded and patients with new infections (determined by PCR) were considered successes at the first time point that this outcome was recorded, but were excluded thereafter.

At Day 14, 16/553 patients were non-evaluable in the CDA group: $1(0.2 \%)$ earlier new infection, $5(0.9 \%)$ without PCR at Day 14 , and 10 (1.8\%) for "other" reasons. In the CPG-DDS group, 10/259 patients were non-evaluable: $3(1.2 \%)$ earlier new infection, $5(1.9 \%)$ without PCR at Day 14 , and $2(0.8 \%)$ "other."

At Day 28, 86/553 patients were non-evaluable in the CDA group: 62 (11.2\%) earlier new infections, $4(0.7 \%)$ without PCR at Day 28 , and 16 (2.9) "other". In the CPG-DDS group, $47 / 259$ patients were non-evaluable: 38 (14.7\%) earlier new infections, 5 (1.9\%) without PCR at Day 28 , and 4 (1.5\%) "other."

\$In the ITT analysis, patients with missing data were treated as failures. Patients with new infections (determined by PCR) were considered successes at the first time point that this outcome was recorded and as failures thereafter. 

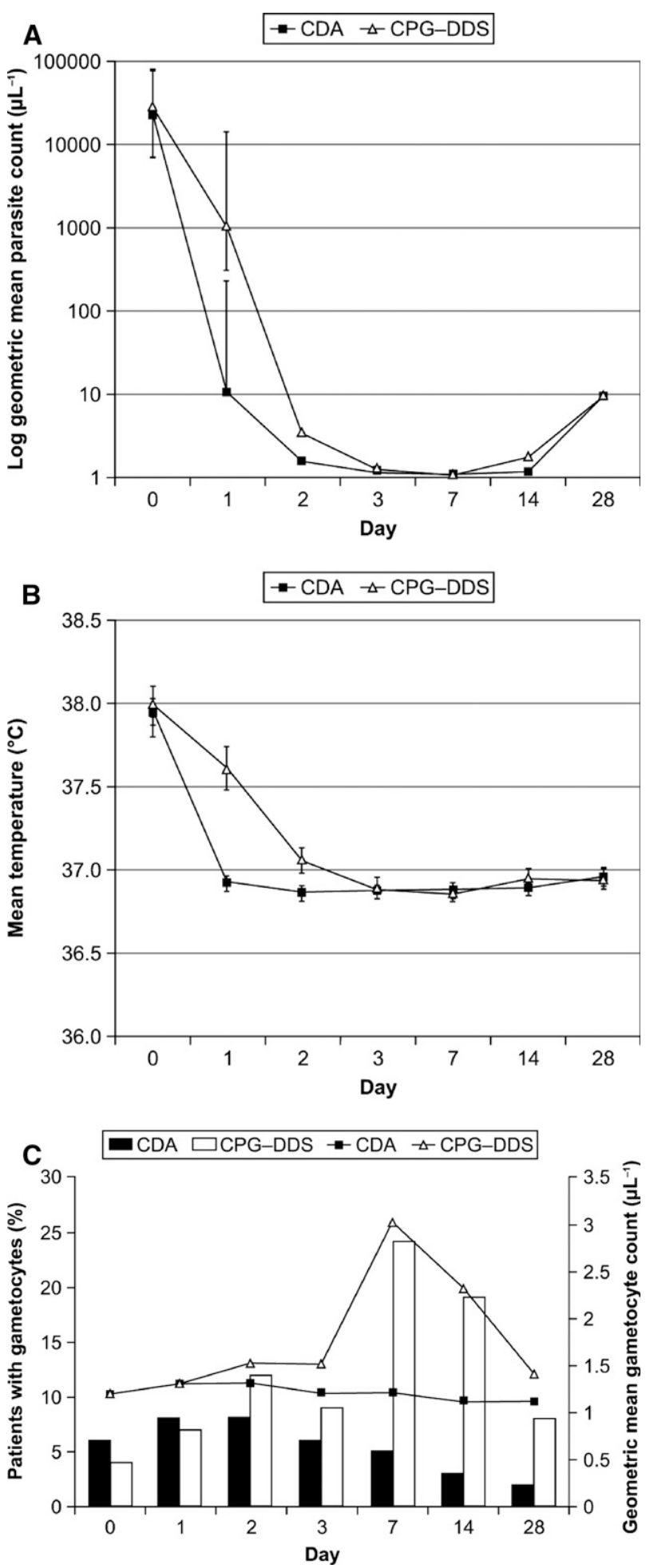

FIGURE 2. The effect of CDA and CPG-DDS on: $\mathbf{A}$, mean parasite load ( \pm interquartile range); $\mathbf{B}$, mean temperature $( \pm 95 \% \mathrm{CI})$; and $\mathbf{C}$, the percentage of patients that were gametocytemic (bars) and geometric mean gametocyte count (lines). NB: error ranges are not shown for gametocyte data as lower and upper quartiles were all zero at every time point because over $75 \%$ of subjects had no gametocytes.

91.9 $\mathrm{g} / \mathrm{L}$ versus $95.1 \mathrm{~g} / \mathrm{L}$ for CDA. Hemoglobin concentrations recovered by Day 28 in both treatment groups (Figure $3 \mathrm{~A}$ ).

When assessed by G6PD status, there was no significant difference in hemoglobin concentrations over time between
CDA and CPG-DDS in G6PD normal, heterozygous, or deficient patients (Figure 3B, C, and D). In G6PD-deficient patients, the decrease in hemoglobin was greater than for G6PD-normal and heterozygous female patients (Figure 3D). In G6PD-deficient patients, the minimum hemoglobin concentration was at Day 7 for both treatment groups, $76.3 \mathrm{~g} / \mathrm{L}$ (95\% CI 71.3, 81.4) with CDA and $81.5 \mathrm{~g} / \mathrm{L}$ (95\% CI 72.4, 90.7) with CPG-DDS.

Adjustment for treatment, age, baseline parasitemia, center and actual dapsone dose showed a significantly greater mean decrease in hemoglobin at Day 7 with CDA $(14.1 \mathrm{~g} / \mathrm{L})$ versus CPG-DDS (12.0 g/L; 95\% CI 0.4, 3.9; $P=0.016)$. At Day 3 , however, the mean hemoglobin decease was significantly greater with CPG-DDS (12.7 g/L) versus CDA (10.5 g/L; $95 \%$ CI $1.0,3.5 ; P<0.001)$. An analysis by G6PD genotype showed that these differences were only significant in G6PDnormal patients (CDA - CPG-DDS): Day $3(-1.77 \mathrm{~g} / \mathrm{L} ; 95 \%$ CI $-3.0,-0.5 ; P=0.005)$ and Day $7(1.83 \mathrm{~g} / \mathrm{L} ; 95 \%$ CI 0.1 , $3.5 ; P=0.033)$. There was no significant difference in adjusted mean change in hemoglobin versus baseline between the treatment groups for G6PD-deficient patients or heterozygous females.

Decreases in hemoglobin of $\geq 20 \mathrm{~g} / \mathrm{L}$ versus baseline occurred in $168 / 600(28.0 \%)$ patients in the CDA group and $98 / 292(33.6 \%)$ in the CPG-DDS group overall. In the CDA group, a $\geq 20 \mathrm{~g} / \mathrm{L}$ hemoglobin drop occurred in $96 / 448$ (21\%) G6PD-normal patients, 30/70 (43\%) heterozygous females, and 30/44 (68\%) G6PD-deficient patients. Corresponding results for CPG-DDS were 63/221 (29\%), 18/37 (49\%), and $15 / 24(63 \%)$, respectively.

There were no major differences between treatment groups for hematocrit or red blood cell (RBC) count. Platelet count improved for both treatment groups, with no remarkable differences between groups. Decreases in WBC count were observed in both treatment groups following the start of dosing and continuing through Day 28.

Clinical chemistry assessments. Alanine transaminase (ALT) elevations of clinical significance occurred in 18/600 (3\%) patients in the CDA group and 4/292 (1\%) in the CPG-DDS group. In most cases ALT elevations were transient and had resolved by Day 28. Two subjects with ALT elevations in the CDA group were diagnosed with hepatitis A. There were no other notable changes in clinical chemistry values.

\section{DISCUSSION}

Although we showed non-inferiority (and superiority) of CDA over CPG-DDS for the primary endpoint, the PCRcorrected Day 28 parasitologic cure rates in the PP population were lower than anticipated; $89.1 \%$ for CDA and $83.0 \%$ for CPG-DDS. A companion Phase III trial conducted at sites in Burkina Faso, Ghana, Kenya, Nigeria, and Tanzania reported Day 28 PCR-corrected parasitologic cure rates (PP population) of $94.1 \%$ (703/747) for CDA versus $97.4 \%$ (369/379) for the comparator AL. ${ }^{19}$ In both studies, the cure rates for CDA fell short of the WHOrecommended average cure rate of $95 \%$ for any new antimalarial medicine. ${ }^{14}$

The lower than expected efficacy of CDA was probably not caused by drug resistance. Resistance to CPG-DDS is thought to require a quadruple $p f d h f r$ mutation at codons $108,51,59$, and $164 .{ }^{26}$ This mutation has been detected rarely in Africa, 
TABLE 4

Adequate clinical and parasitologic response uncorrected (ACPR), and PCR-corrected (ACPRp)*

\begin{tabular}{|c|c|c|c|c|c|}
\hline \multirow[b]{2}{*}{ Analysis } & \multirow[b]{2}{*}{ Outcome, $\mathrm{n} / N(\%)$} & \multicolumn{2}{|c|}{ PP population $\dagger$} & \multicolumn{2}{|c|}{ ITT population $\dagger$} \\
\hline & & CDA & CPG-DDS & $\operatorname{CDA}(N=600)$ & CPG-DDS $(N=292)$ \\
\hline \multirow[t]{5}{*}{ Day 14} & Early treatment failure & $11 / 548(2)$ & 27/257 (11) & $24(4)$ & $39(13)$ \\
\hline & Late clinical failure & $1 / 546(<1)$ & $2 / 258(<1)$ & $8(1)$ & $3(1)$ \\
\hline & Late parasitological failure & $18 / 548(3)$ & $12 / 257(5)$ & $16(3)$ & $18(6)$ \\
\hline & $\mathrm{ACPR}$ & $513 / 543(94)$ & $215 / 256(84)$ & $552(92)$ & $232(79)$ \\
\hline & ACPRp§ & $524 / 543(97)$ & $219 / 256(86)$ & $563(94)$ & $237(81)$ \\
\hline \multirow[t]{4}{*}{ Day 28} & Late clinical failure & 65/551 (12) & $36 / 258(14)$ & $79(13)$ & $45(15)$ \\
\hline & Late parasitological failure & $157 / 548(29)$ & $67 / 257(26)$ & $165(28)$ & $75(26)$ \\
\hline & ACPR & $311 / 544(57)$ & $126 / 256(49)$ & $332(55)$ & $133(46)$ \\
\hline & ACPRp§ & 484/544 (89) & 201/256 (79) & $519(87)$ & $217(74)$ \\
\hline
\end{tabular}

*ACPR $=$ adequate clinical and parasitologic response PCR $=$ polymerase chain reaction; $\mathrm{PP}=$ per-protocol; $\mathrm{ITT}=$ intent-to-treat; $\mathrm{CDA}=$ chlorproguanil-dapsone-artesunate CPG-DDS = chlorproguanil-dapsone.

$\dagger$ For the PP population, patients with missing data or indeterminate results were excluded-the denominator shows the number of evaluable patients. For the ITT population, patients with missing or indeterminate PCR results were treated as failures. For ACPRp, in the PP and ITT analysis, patients with new infections were considered successes at that time point. However, at subsequent time points, the PP analyses considered these patients as missing and the ITT analysis assumed that they were failures.

\$Early treatment failure rates are the same at Day 28 .

$\S$ ACPRp is corrected for reinfection.

though there are reports from Kenya and at "hot-spots" in Uganda. ${ }^{27,28}$ A limitation of both CDA Phase III studies was that drug resistance assays were not performed.

Two other recent studies also reported unsatisfactory efficacy with CPG-DDS plus artesunate (A) given concomitantly. The Day 28 ACPRp with CPG-DDS+A at two sites in Rwanda was $71.9 \%$ (274/381) overall, versus 63.2\% (247/391) for amodiaquine $+\mathrm{SP}^{29} \mathrm{~A}$ trial in Ghana reported PCR-corrected Day 28 parasitological and clinical cure rates (PP population) of $86.3 \%(138 / 160)$ for CPG-DDS + A, 86.2\% (131/152) for $\mathrm{AL}$, and $93.4 \%(141 / 151)$ for amodiaquine $+\mathrm{A}(P=0.08){ }^{30}$ Neither of these studies has reported pfdhfr mutation frequencies, though the authors of the Rwandan study state that a later analysis is planned. ${ }^{29}$

There was no difference in re-infection rates between CDA and CPG-DDS. There was a relatively high intensity of transmission in specific sites in our study. Overall, the re-infection rate was $32 \%$ (281/892), ranging from $45 \%$ (162/360) in Ouagadougou to $0 \%(0 / 46)$ in Jos and (0/4) Lagos. These results were expected given the rapidly eliminated drug combinations; the parasitocidal activity of CPG-DDS is 3-10 days, depending on parasite strain. ${ }^{31}$ In fact, the rationale for the CDA combination was to cure the initial infection, while avoiding prolonged drug concentrations that could select for resistant parasites after the elimination of artesunate.

TABLE 5

Hemoglobin safety composite endpoint occurrences (hemoglobin decrease of $\geq 40 \mathrm{~g} / \mathrm{L}$ or $\geq 40 \%$ vs. baseline or hemoglobin $<50 \mathrm{~g} / \mathrm{L}$ or blood transfusion) by G6PD genotype and phenotype (ITT population)*

\begin{tabular}{|c|c|c|}
\hline Population, $\mathrm{n} / N(\%)$ & $\operatorname{CDA}(N=600)$ & CPG-DDS $(N=292)$ \\
\hline \multicolumn{3}{|l|}{ G6PD genotype } \\
\hline Normal & $4 / 448(<1)$ & 6/221 (3) \\
\hline Female heterozygous & $0 / 70$ & $1 / 37(3)$ \\
\hline G6PD deficient $\dagger$ & $13 / 44(30)$ & $7 / 24(29)$ \\
\hline Missing & $3 / 38(8)$ & $1 / 10(10)$ \\
\hline \multicolumn{3}{|l|}{ G6PD phenotype } \\
\hline Normal & $3 / 406(<1)$ & $5 / 202(2)$ \\
\hline Intermediate & $1 / 47(2)$ & $1 / 26(4)$ \\
\hline Deficient & $11 / 48(23)$ & $5 / 18(28)$ \\
\hline Missing & $5 / 99(5)$ & $4 / 46(9)$ \\
\hline Total & $20 / 600(3)$ & $15 / 292(5)$ \\
\hline
\end{tabular}

Parasite clearance and fever resolution were more rapid with CDA versus CPG-DDS. There was also a lower proportion of early treatment failure in the CDA group (2\%) versus CPG-DDS (11\%). Artemisinins are gameotocytocidal, ${ }^{9}$ and gametocytes were more effectively suppressed with CDA versus CPG-DDS. These observations clearly show the advantages of artesunate in the combination and support the current WHO recommendations for ACT use throughout Africa. ${ }^{14}$

The major safety finding of this study concerned the hemolytic effects of both treatments in G6PD-deficient patients. Overall, 53/414 (13\%) males were G6PD hemizygous and 15/430 (3\%) females were G6PD homozygous (i.e., G6PD deficient). Furthermore, 107/453 (24\%) females were G6PD heterozygous. The majority of malaria patients in Africa would be unaware of their G6PD status and G6PD testing is not generally available.

This study was not powered to compare hematologic safety in G6PD-deficient versus G6PD-normal individuals. However, we defined a composite hemoglobin safety endpoint to identify and group those drops in hemoglobin that would usually require medical intervention. Access to hospital and clinic facilities are often limited in sub-Saharan Africa, and any occurrence of the composite endpoint would represent an appreciable risk for an unfavorable patient outcome. Occurrences of the hemoglobin safety endpoint were 20/68 (29\%) in G6PD-deficient patients versus 10/669 $(1.5 \%)$ in G6PD-normal patients overall. There was no difference in hemolytic risk between treatment groups. Logistic regression identified G6PD deficiency versus G6PD normal as the only significant risk factor for the hemoglobin safety endpoint (odds ratio 40.0; $95 \%$ CI 16.1, 99.4; $P<$ 0.001). G6PD phenotyping detected all but one patient who experienced the hemoglobin safety endpoint; a heterozygous female.

The results for the companion Phase III trial of CDA versus AL also showed an unacceptable risk of hemolytic adverse events for G6PD-deficient patients in the CDA arm. ${ }^{19}$ In that study, 28/80 (35\%) of G6PD-deficient patients in the CDA arm had an occurrence of the hemoglobin safety endpoint versus 0/36 with AL. The main risk factors associated with the hemoglobin safety endpoint were G6PD deficient versus G6PD normal (OR 16.3; 95\% CI 8.6, 31.2) and CDA treatment versus AL (OR 5.1; $95 \%$ CI 2.1, 12.4). 
A

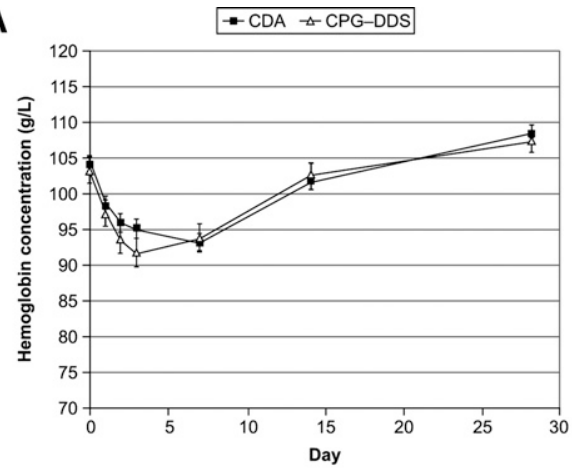

C

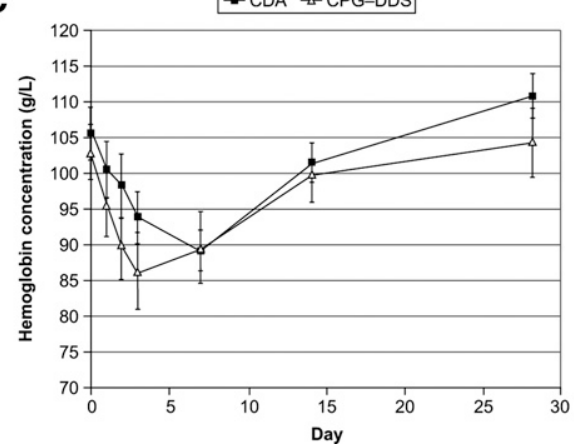

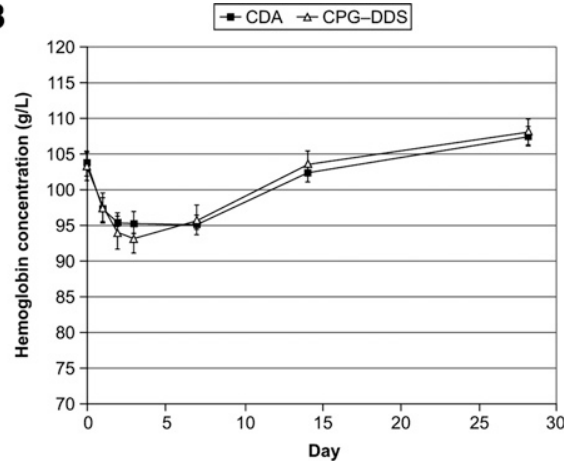

D

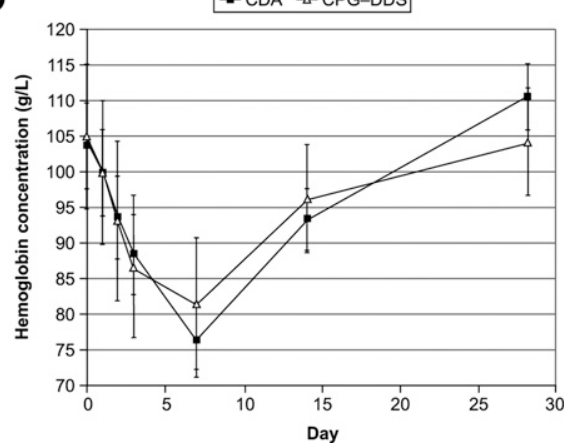

Figure 3. Mean hemoglobin $( \pm 95 \%$ CI) over time with CDA and CPG-DDS in the intent-to-treat (ITT) population for: $\mathbf{A}$, all patients; and by G6PD genotype: B, normal; $\mathbf{C}$, female heterozygous; and $\mathbf{D}$, deficient (male hemizygous and female homozygous). Day 0 are screening (baseline) values.

CDA was envisaged as an affordable artemisinin-based combination therapy for use in Africa. However, given the high prevalence of G6PD-deficiency in sub-Saharan Africa, and the current availability of alternative ACTs, the risk:benefit balance of treating mild-to-moderate malaria with CDA in this setting would be unacceptable. Consequently, the CDA development program has been discontinued and CPG-DDS licenses have been withdrawn voluntarily.

Previous studies of CPG-DDS did not prospectively include G6PD genotyping for all subjects. The major CPG-DDS safety study by Alloueche and others ${ }^{4}$ retrospectively genotyped some patients with hemoglobin drops of $\geq 20 \mathrm{~g} / \mathrm{L}$ versus baseline and compared them to controls. However, because of sample bias and hemoglobin readings not being collected at the appropriate time points, the hemolytic potential of CPG-DDS in G6PD-deficient patients could not be fully determined. ${ }^{16} \mathrm{~A}$ recent open randomized trial in patients with uncomplicated P. falciparum conducted in Rwanda, compared CPG-DDS + A with amodiaquine + SP and included a secondary analysis for the risk of severe anemia in patients with G6PD deficiency. ${ }^{29,32}$ Results were analyzed using mean packed cell volume (PCV) and so cannot be compared directly with the results reported here. However, as in the current study of the CDA fixed-dose combination, CPG-DDS + A was associated with increased hemolysis and a greater risk of blood transfusion in G6PDdeficient versus G6PD-normal patients. ${ }^{32}$

There were some limitations in the hematologic data collected during this study. Methemoglobin levels were measured for only five patients in each treatment arm at screening. A clinic-based methemoglobin test was not available at the start of the study and data could not be routinely collected at the study sites. Reticulocyte absolute values and percentages were collected, but methods were not automated and there was no quality control process. Meaningful analysis of these data was not possible and they are not presented. Unfortunately, delays in reporting G6PD phenotype and genotype meant that these data were not available to the IDMC until after recruitment was complete and so could not be linked to hematological adverse events.

We believe this to be the first Phase III clinical trial program in any field to prospectively include G6PD genotyping. This work answers the important question of whether the G6PDrelated hemolysis observed with CPG-DDS is exacerbated in the presence of artesunate. In fact, the hemolytic adverse effects of CDA and CPG-DDS in this work are very similar overall and in the G6PD-deficient sub-population. This is reassuring regarding the use of artesunate in other combinations, but raises questions regarding the risk:benefit of other potentially oxidative agents when used in G6PD-deficient populations.

Received June 22, 2009. Accepted for publication August 17, 2009.

Acknowledgments: Naomi Richardson of Magenta Communications Ltd. developed a first draft of this paper from the approved study report and collated author contributions and was funded by GlaxoSmithKline. The authors thank all those who were involved in the clinical trial teams at Ouagadougou, Burkina Faso; Kumasi, Ghana; Doneguebougou and Banambani, Mali; Ile-Ife, Jos, and Lagos, Nigeria.

Financial support: Chlorproguanil-dapsone-artesunate (CDA) Joint Development Team. The development of CDA was conducted through a public-private partnership within a development agreement between the Medicines for Malaria Venture, the World Health Organization Special Program for Research and Training in Tropical Medicine and GlaxoSmithKline PLC. Liverpool School of Tropical Medicine, Liverpool University, and the London School of Hygiene and Tropical Medicine joined the development team as academic partners. 
Disclosure: Li-ean Goh sadly died while this paper was in preparation; the paper has been reviewed and approved by her colleagues (Allan Pamba, Emma Harrell, and Nick Carter) on her behalf. Allan Pamba, Emma Harrell, and Nick Carter are all current employees of GlaxoSmithKline. Stephan Duparc is a former employee of GlaxoSmithKline and a current employee of Medicines for Malaria Venture. Stephen A. Ward was a member of the Lapdap and CDA product development team. A committee representing members of the CDA Joint Development Team and study site Principal Investigators developed the protocol. GlaxoSmithKline conducted the study, collected and analyzed data. All authors had access to the primary data and take responsibility for data reporting accuracy and completeness. The corresponding author had responsibility for the final decision to submit for publication.

Authors' addresses: Alfred B. Tiono, Centre National de Recherche et de Formation sur le Paludisme, Ministère de la Santé, 01 BP 2208, Ouagadougou 01, Burkina Faso, Tel: +226-503-24695, Fax: +226-50305220 or +226-503-10477, E-mail: t.alfred@fasonet.bf. Alassane Dicko, Malaria Research and Training Centre and Department of Public Health, Faculty of Medicine, Pharmacy and Dentistry, University of Bamako, P.O. Box 1805, Bamako, Mali, Tel: +223-2022-8109/+2236674-2296, Fax: +223-2022-4987, E-mails: adicko@mrtcbko.org or adicko@icermali.org. Dennis A. Ndububa, Department of Medicine, College of Health Sciences, Obafemi Awolowo University, Ile Ife 220005, Nigeria, Tel: +234-803-719-4917, Fax: +234-803-980-3828, E-mail: dennisandububa@yahoo.co.uk. Tsiri Agbenyega, Komfo Anokye Teaching Hospital, P.O. Box 1934, Kumasi-Ashanti, Ghana, Tel: +233-516-0302, Fax: +233-516-0302/0137, E-mail: tsiri@ghana .com. Simon Pitmang, Department of Medicine, Plateau State Specialist Hospital, Jos, Plateau State, PMB 2113, Nigeria, Tel: +234734-64031, E-mail: pitmangsl@yahoo.co.uk. Jacob Awobusuyi, Lagos State University Teaching Hospital, Department of Medicine, 1-4 Oba Akinjobi Street, Ikeja, Lagos, Nigeria, Tel: +234-802-304-6830, E-mail: awojaco@yahoo.com. Allan Pamba, ID-MDC Diseases of the Developing World, GlaxoSmithKline, Greenford Road, Greenford, Middlesex, UB6 0HE, United Kingdom, Tel: +44-208-422-3434, Fax: +44-208-423-4401, E-mail: allan.pamba@GSK.com. Stephan Duparc, Medicines for Malaria Venture (MMV), International Center Cointrin (ICC) Building, 20, rte de Pré-Bois, Block G, 3rd Floor, 1215Geneva15,Switzerland,Tel:+41-22-799-4060,Fax:+41-22-799-4061, E-mail: duparcs@mmv.org. Li-Ean Goh, please refer any correspondence to Allan Pamba. Emma Harrell, Global Clinical Safety and Pharmacovigilance, GlaxoSmithKline, New Frontiers Science Park, Third Avenue, Harlow, Essex, CM19 5AW, United Kingdom, Tel: +44-1279-622000, Fax: +44-1279-644983, E-mail: emma.2.harrell@ gsk.com. Nick Carter, ID-MDC Diseases of the Developing World, GlaxoSmithKline, Greenford Road, Greenford, Middlesex, UB6 0HE, United Kingdom, Tel: +44-208-422-3434, Fax: +44-208-423-4401, E-mail: nick.s.carter@gsk.com. Stephen A. Ward, Liverpool School of Tropical Medicine, Pembroke Place, Liverpool, L3 5QA, United Kingdom, Tel: +44-151-705-3286, Fax: +44-151-705-3371, E-mail: saward@liverpool.ac.uk. Brian Greenwood, Department of Infectious and Tropical Diseases, London School of Hygiene and Tropical Medicine, Keppel Street, London, WC1E 7HT, United Kingdom, Tel: +44-20-7299-4707, Fax: +44-20-7299-4720, E-mail: brian.greenwood@ lshtm.ac.uk.Peter A. Winstanley, School of Clinical Sciences, University of Liverpool, Liverpool, United Kingdom, Tel: +44-151-706-4157 Fax: +44-151-706-4072, E-mail: p.a.winstanley@liverpool.ac.uk.

\section{REFERENCES}

1. World Health Organization, 2008. World Malaria Report. Geneva: World Health Organization. Available at: http://www.who.int/ malaria/wmr2008/malaria2008.pdf. Accessed April 12, 2009.

2. Campbell CC, 2008. Halting the toll of malaria in Africa. Am J Trop Med Hyg 78: 851-853.

3. Winstanley P, 2001. Chlorproguanil-dapsone (LAPDAP) for uncomplicated falciparum malaria. Trop Med Int Health 6: 952-954.

4. Alloueche A, Bailey W, Barton S, Bwika J, Chimpeni P, Falade CO, Fehintola FA, Horton J, Jaffar S, Kanyok T, Kremsner PG, Kublin JG, Lang T, Missinou MA, Mkandala C, Oduola AM, Premji Z, Robertson L, Sowunmi A, Ward SA, Winstanley PA, 2004. Comparison of chlorproguanil-dapsone with sulfadoxine- pyrimethamine for the treatment of uncomplicated falciparum malaria in young African children: double-blind randomised controlled trial. Lancet 363: 1843-1848.

5. Price RN, Nosten F, Luxemburger C, van Vugt M, Phaipun L, Chongsuphajaisiddhi T, White NJ, 1997. Artesunate/mefloquine treatment of multi-drug resistant falciparum malaria. Trans $R$ Soc Trop Med Hyg 91: 574-577.

6. van Vugt M, Looareesuwan S, Wilairatana P, McGready R, Villegas L, Gathmann I, Mull R, Brockman A, White NJ, Nosten F, 2000. Artemether-lumefantrine for the treatment of multidrugresistant falciparum malaria. Trans $R$ Soc Trop Med Hyg 94: $545-548$.

7. Alin MH, Bjorkman A, 1994. Concentration and time dependency of artemisinin efficacy against Plasmodium falciparum in vitro. Am J Trop Med Hyg 50: 771-776.

8. Angus BJ, Thaiaporn I, Chanthapadith K, Suputtamongkol Y, White NJ, 2002. Oral artesunate dose-response relationship in acute falciparum malaria. Antimicrob Agents Chemother 46: 778-782.

9. Dutta GP, Bajpai R, Vishwakarma RA, 1989. Artemisinin (qinghaosu)-a new gametocytocidal drug for malaria. Chemotherapy 35: 200-207.

10. Barnes KI, Durrheim DN, Little F, Jackson A, Mehta U, Allen E, Dlamini SS, Tsoka J, Bredenkamp B, Mthembu DJ, White NJ, Sharp BL, 2005. Effect of artemether-lumefantrine policy and improved vector control on malaria burden in KwaZulu-Natal, South Africa. PLoS Med 2: e330.

11. Bousema JT, Schneider P, Gouagna LC, Drakeley CJ, Tostmann A, Houben R, Githure JI, Ord R, Sutherland CJ, Omar SA, Sauerwein RW, 2006. Moderate effect of artemisinin-based combination therapy on transmission of Plasmodium falciparum. J Infect Dis 193: 1151-1159.

12. Hallett RL, Sutherland CJ, Alexander N, Ord R, Jawara M, Drakeley CJ, Pinder M, Walraven G, Targett GA, Alloueche A, 2004. Combination therapy counteracts the enhanced transmission of drug-resistant malaria parasites to mosquitoes. Antimicrob Agents Chemother 48: 3940-3943.

13. Price RN, Nosten F, Luxemburger C, ter Kuile FO, Paiphun L, Chongsuphajaisiddhi T, White NJ, 1996. Effects of artemisinin derivatives on malaria transmissibility. Lancet 347: 16541658.

14. World Health Organization, 2006. Guidelines for the Treatment of Malaria. Geneva: World Health Organization. Available at: http://malaria.who.int/docs/TreatmentGuidelines2006.pdf. Accessed August 22, 2008.

15. Wootton DG, Opara H, Biagini GA, Kanjala MK, Duparc S, Kirby PL, Woessner M, Neate C, Nyirenda M, Blencowe H, Dube-Mbeye Q, Kanyok T, Ward S, Molyneux M, Dunyo S, Winstanley PA, 2008. Open-label comparative clinical study of chlorproguanil-dapsone fixed dose combination (Lapdap) alone or with three different doses of artesunate for uncomplicated Plasmodium falciparum malaria. PLoS One 3: e1779.

16. World Health Organization, 2004. Review of the safety of chlorproguanil-dapsone in the treatment of uncomplicated falciparum malaria in Africa. Report of a Technical Consultation Convened by WHO. Geneva: World Health Organization. Available at: http://www.who.int/malaria/docs/LapDap.pdf. Accessed July 23, 2008.

17. Beutler E, Duparc S, 2007. Glucose-6-phosphate dehydrogenase deficiency and antimalarial drug development. Am J Trop Med Hyg 77: 779-789.

18. Beutler E, Kuhl W, Vives-Corrons JL, Prchal JT, 1989. Molecular heterogeneity of glucose-6-phosphate dehydrogenase A. Blood 74: $2550-2555$

19. Premji Z, Umeh R, Owusu-Agyei S, Esamai F, Ezedinachi E, Oguche S, Borrmann S, Sowunmi A, Duparc S, Kirby P, Pamba A, Kellam L, Guiguemdé R, Greenwood B, Ward S, Winstanley P, 2009. Chlorproguanil-dapsone-artesunate versus artemetherlumefantrine: a randomized, double-blind phase III trial in African children and adolescents with uncomplicated Plasmodium falciparum malaria. PLoS One 4: e6682.

20. World Health Organization, 2003. Assessment and Monitoring of Antimalarial Drug Efficacy for the Treatment of Uncomplicated Falciparum Malaria. (WHO/HTM/RBM/2003.50). Geneva: World Health Organization. Available at: http://www.who.int/ malaria/docs/ProtocolWHO.pdf. Accessed July 6, 2008. 
21. Cattamanchi A, Kyabayinze D, Hubbard A, Rosenthal PJ, Dorsey $\mathrm{G}, 2003$. Distinguishing recrudescence from reinfection in a longitudinal antimalarial drug efficacy study: comparison of results based on genotyping of $m s p-1, m s p-2$, and glurp. Am J Trop Med Hyg 68: 133-139.

22. Medicines for Malaria Venture, World Health Organization, 2008. Methods and Techniques for Clinical Trials on Anti-Malarial Drug Efficacy: Genotyping to Identify Parasite Populations. Geneva: World Health Organization. Available at: http://www who.int/malaria/docs/drugresistance/MalariaGenotyping.pdf. Accessed July 25, 2008.

23. Nafa K, Reghis A, Osmani N, Baghli L, Ait-Abbes H, Benabadji M, Kaplan JC, Vulliamy T, Luzzatto L, 1994. At least five polymorphic mutants account for the prevalence of glucose-6-phosphate dehydrogenase deficiency in Algeria. Hum Genet 94: 513-517.

24. Poggi V, Town M, Foulkes NS, Luzzatto L, 1990. Identification of a single base change in a new human mutant glucose-6-phosphate dehydrogenase gene by polymerase-chain-reaction amplification of the entire coding region from genomic DNA. Biochem J 271: 157-160.

25. Samilchuk E, D'Souza B, Al-Awadi S, 1999. Population study of common glucose-6-phosphate dehydrogenase mutations in Kuwait. Hum Hered 49: 41-44.

26. Krudsood S, Imwong M, Wilairatana P, Pukrittayakamee S, Nonprasert A, Snounou G, White NJ, Looareesuwan S, 2005. Artesunate-dapsone-proguanil treatment of falciparum malaria: genotypic determinants of therapeutic response. Trans $R$ Soc Trop Med Hyg 99: 142-149.

27. Lynch C, Pearce R, Pota H, Cox J, Abeku TA, Rwakimari J, Naidoo I, Tibenderana J, Roper C, 2008. Emergence of a $d h f r$ mutation conferring high-level drug resistance in Plasmodium falciparum populations from southwest Uganda. J Infect Dis 197: 1598-1604.

28. Hamel MJ, Poe A, Bloland P, McCollum A, Zhou Z, Shi YP, Ouma P, Otieno K, Vulule J, Escalante A, Udhayakumar V, Slutsker L, 2008. Dihydrofolate reductase I164L mutations in Plasmodium falciparum isolates: clinical outcome of 14 Kenyan adults infected with parasites harbouring the $\mathrm{I} 164 \mathrm{~L}$ mutation. Trans $R$ Soc Trop Med Hyg 102: 338-345.

29. Fanello CI, Karema C, Ngamije D, Uwimana A, Ndahindwa V, Van Overmeir C, Van Doren W, Curtis J, D’Alessandro U, 2008. A randomised trial to assess the efficacy and safety of chlorproguanil/dapsone + artesunate for the treatment of uncomplicated Plasmodium falciparum malaria. Trans $R$ Soc Trop Med Hyg 102: 412-420.

30. Owusu-Agyei S, Asante KP, Owusu R, Adjuik M, Amenga-Etego S, Dosoo DK, Gyapong J, Greenwood B, Chandramohan D, 2008. An open label, randomised trial of artesunate + amodiaquine, artesunate + chlorproguanil-dapsone and artemetherlumefantrine for the treatment of uncomplicated malaria. PLoS One 3: e2530.

31. Simpson JA, Hughes D, Manyando C, Bojang K, Aarons L, Winstanley P, Edwards G, Watkins WA, Ward S, 2006. Population pharmacokinetic and pharmacodynamic modelling of the antimalarial chemotherapy chlorproguanil/dapsone. Br J Clin Pharmacol 61: 289-300.

32. Fanello CI, Karema C, Avellino P, Bancone G, Uwimana A, Lee SJ, d'Alessandro U, Modiano D, 2008. High risk of severe anaemia after chlorproguanil-dapsone + artesunate antimalarial treatment in patients with G6PD (A-) deficiency. PLoS One 3: e4031. 